\title{
On the detection of characteristic optical emission from electronically coupled nanoemitters
}

\author{
David S. Bradshaw, Jack S. Ford and David L. Andrews \\ School of Chemistry, University of East Anglia, Norwich Research Park, Norwich NR4 7TJ, U. K.
}

\begin{abstract}
Optical emission from an electronically coupled pair of nanoemitters is investigated, in a new theoretical development prompted by experimental work on oriented semiconductor polymer nanostructures. Three physically distinct mechanisms for photon emission by such a pair, positioned in the near-field, are identified: emission from a pairdelocalized exciton state, emission that engages electrodynamic coupling through quantum interference, and correlated photon emission from the two components of the pair. Each possibility is investigated, in detail, by examination of the emission signal via explicit coupling of the nanoemitter pair with a photodetector, enabling calculations to give predictive results in a form directly tailored for experiment. The analysis incorporates both near- and far-field properties (determined from the detector-pair displacement), so that the framework is applicable not only to a conventional remote detector, but also a near-field microscope setup. The results prove strongly dependent on geometry and selection rules. This work paves the way for a broader investigation of pairwise coupling effects in the optical emission from structured nanoemitter arrays.
\end{abstract}

Keywords: nanophotonics, optical emission, quantum interference, nanoemitters, near field, far field, energy transfer, nanoscale materials, quantum electrodynamics

\section{INTRODUCTION}

Optical nanoantennas represent the forefront of modern miniaturization of technology originating in the radio-frequency domain. ${ }^{1-3}$ Many of the most attractive modern systems operate on the basis of the distinctive dispersion properties and intense plasmonic response of metallic nanoparticles at optical frequencies, and one of the most appealing facets of such systems is their capacity to steer directional emission through nanoantenna-emitter coupling - especially by interface with gold nanoantennas. ${ }^{4-17}$ Alongside these developments, a range of other plasmonic and dielectric methods have also been demonstrated to provide for enhanced rates of fluorescence ${ }^{18-22}$ and resonance energy transfer, ${ }^{23,24}$ from and between nanoscale components. Moreover, there is recent theory work that has identified new opportunities for nonlinear optical techniques to improve data capture in fluorescence microscopy and imaging. ${ }^{25}$

Rapidly escalating research in this whole area has, until now, mostly focused either upon the action and activity of individual optical components, or the collective response of extended one- or two-dimensional arrays. ${ }^{26-31}$ It is therefore timely, and of considerable interest, to consider in more detail what kinds of effect might arise from the coupling of nanoemitters paired in close proximity. ${ }^{32-34}$ The theory work that follows, building on earlier calculations, ${ }^{35}$ aims to identify distinctive electromagnetic coupling features in the optical emission from dielectric nanoparticle pairs, focusing on the interplay between electronic and local geometric configurations where phase properties can be anticipated to play a prominent role. New results, recently derived and now reported here, explicitly take account of the coupling between the nanoemitters and the detector. As such, the analysis offers a robust means to predict the full intensity and phase properties of the signal fluorescence. This is a photonic formulation directly tailored for the identification of both nearand far-field properties, enabling the results to be applied not only to a conventional remote detector, but also to measurements made with a near-field microscope. 


\section{THE TWIN EMITTER-DETECTOR SYSTEM}

First we consider a system comprising two emitters and a photodetector. Although the quantum electrodynamical formulation of theory we are to provide is amenable to application to a wide range of physically very different systems, here we shall focus on a pair of emitters which are molecules - in due course also assuming that they are separated by a distance that is much less than the wavelength of light in the visible region, such that the coupling between them can be treated as a near-field interaction. The detector is assumed to comprise a suitably wavelength-specific absorbing component on a support that is conceivably movable in the near-field, as for example on an AFM tip. However, no specific restriction is placed on the detector-pair separation, which may equally be in the far-field (remote detector) regime.

An electrodynamical process evolving from an initial quantum state $i$ to a final state $f$, has an observable rate determined (according to the Fermi rule) by the square modulus of a quantum amplitude, $M$, the latter as given by the following perturbation expansion;

$$
M=\sum_{r} \frac{\left\langle f\left|H_{\text {int }}\right| r\right\rangle\left\langle r\left|H_{\text {int }}\right| i\right\rangle}{\left(E_{i}-E_{r}\right)}+\sum_{r, s, t} \frac{\left\langle f\left|H_{\text {int }}\right| t\right\rangle\left\langle t\left|H_{\text {int }}\right| s\right\rangle\left\langle s\left|H_{\text {int }}\right| r\right\rangle\left\langle r\left|H_{\text {int }}\right| i\right\rangle}{\left(E_{i}-E_{r}\right)\left(E_{i}-E_{s}\right)\left(E_{i}-E_{t}\right)}+\ldots
$$

Here, an interaction Hamiltonian $H_{\text {int }}$ is engaged in each specific molecule-photon interaction, and summations are to be effected over system states $r, s$, $t$, etc. The energy differences that appear in the denominators of equation (1), $E_{i}-E_{r}$ etc., relate to system energies, each term of which can be decomposed into a sum of molecular electronic state energies and overall energy content for the radiation field. The emission process involves nanoemitters which are to be labeled $A$ and $B$ : for emission to occur one or the other will initially occupy an excited molecular state $m$, whilst its neighbor will usually be in its ground state denoted by 0 (we shall briefly consider a strongly-pumped case where both are excited, in Section 3.3); detector $D$ is initially in its similarly-labeled ground state. Once emission is complete, $A$ and $B$ will be in their ground states, and $D$ will have been promoted to an excited state $\gamma$ through the act of detection. The fact that no photons are present in either the initial or final system state is reflected in the inclusion of only even-order terms in equation (1). The energies of these states relate to each other by the relations $\hbar c k=E_{m}^{A}-E_{0}^{A}=E_{m}^{B}-E_{0}^{B}=E_{\gamma}^{D}-E_{0}^{D}$, where $\hbar c k$ is the fluorescence photon energy.

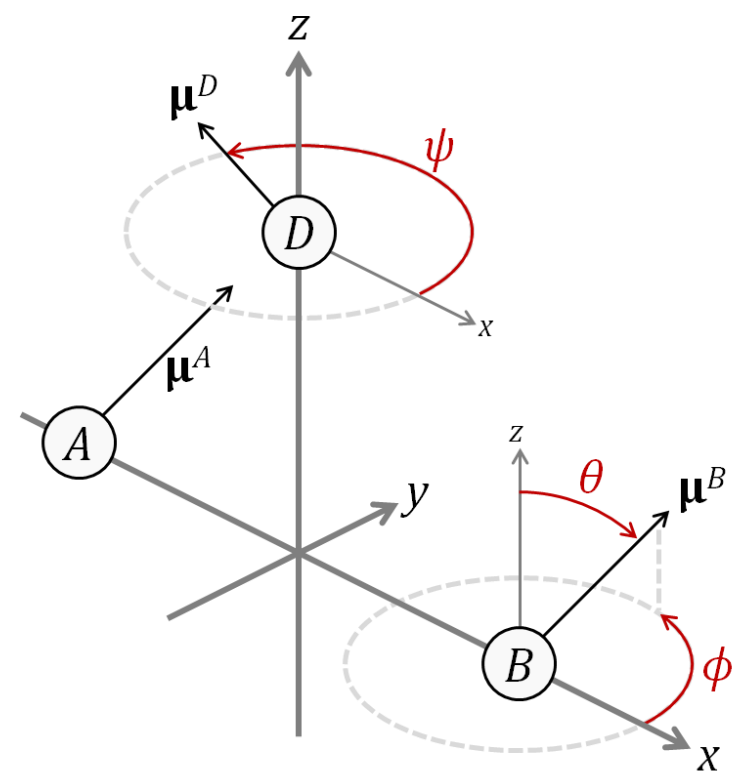

Figure 1. Defining the geometry of the system comprising nanoemitters $A$ and $B$ and detector $D$. 
The sought detector signal for the optical emission is a result of energy being transmitted from the nanoemitter pair to the photodetector. It is possible to rigorously describe both the electromagnetic coupling that operates between each emitter and the detector, and also the coupling between the emitters (involved in producing distinctive features in the pair fluorescence) in a single consistent quantum framework. In this sense, the fundamental mechanism for the coupling between any two components entails the transfer of a (virtual) photon. Although our focus is on the delivery of optical energy from an excited emitter (say, the primary nanoemitter $A$ ) to the detector, certain characteristic effects arise from the additional electrodynamic involvement of molecule $B$, positioned in the near-field region of $A$. It is assumed that $A$ and $B$ are equidistant from $D$, as in a standard experimental setup, and also that the two emission dipole moments, i.e. $\boldsymbol{\mu}^{A}$ and $\boldsymbol{\mu}^{B}$, are parallel to each other. Fixing a coordinate origin midway between $A$ and $B$, the position of $D$ serves to define the $z$-axis, as shown in Figure 1. The $\boldsymbol{\mu}^{D}$ vector is limited to the $(x, y)$ plane for simplicity, and the specific orientations of the three transition dipole moments are defined by angles $\theta, \phi$ and $\psi$ as illustrated.

\section{OPTICAL MECHANISMS FOR TWIN-EMITTER ELECTRONIC DECAY}

\subsection{Excitation localized on one emitter}

Consider molecular excitation localized on one of the emitters, $A$. The initial and final system states are given by the Dirac 'kets' $|i\rangle=\left|D_{0}\right\rangle\left|A_{m}, B_{0}\right\rangle$ and $|f\rangle=\left|D_{\gamma}\right\rangle\left|A_{0}, B_{0}\right\rangle$, respectively. Accounting for contributions from both terms in (1), the overall quantum amplitude is given by;

$$
M^{A}=M^{D A}+M^{D A B}+M^{D B A}+M^{B D A}
$$

The leading term, corresponding to the first term of (1), signifies direct coupling of $D$ with $A$. With implied summation over repeated Cartesian indexes, it is expressible as;

$$
M^{D A}=\mu_{i}^{\gamma 0(D)} V_{i j}\left(k ; \mathbf{R}_{D A}\right) \mu_{j}^{0 m(A)}
$$

Here, the superscripts on the transition dipoles designate the nature of the transition, e.g. $\gamma 0$ denotes the transition $\gamma \leftarrow 0$, and $R_{D A}$ is the displacement of $A$ with respect to $D$. There are three fourth-order terms, deriving from the second term of equation (1); these relate to distinct combinations of pairwise coupling, one of which is illustrated by Figure 2. These are the leading contributions to the quantum amplitude with a direct dependence on the relative positioning of the two emitters. Extending the use of superscripts on $M$ to represent intermolecular connectivity, we have; ${ }^{36}$

$$
\left.\begin{array}{l}
M^{D A B}=\mu_{i}^{\gamma 0(D)} V_{i j}\left(k ; \mathbf{R}_{D A}\right) \alpha_{j k}^{0 m(A)}(-k ; 0) V_{k l}\left(0 ; \mathbf{R}_{A B}\right) \mu_{l}^{00(B)} \\
M^{D B A}=\mu_{i}^{\gamma 0(D)} V_{i j}\left(k ; \mathbf{R}_{D B}\right) \alpha_{j k}^{00(B)}(-k ; k) V_{k l}\left(k ; \mathbf{R}_{A B}\right) \mu_{l}^{0 m(A)} \\
M^{B D A}=\mu_{i}^{00(B)} V_{i j}\left(0 ; \mathbf{R}_{D B}\right) \alpha_{j k}^{\gamma 0(D)}(k ; 0) V_{k l}\left(k ; \mathbf{R}_{D A}\right) \mu_{l}^{0 m(A)}
\end{array}\right\}
$$

The intermolecular coupling tensor, $V_{i j}(p ; \mathbf{R})$, which features in equations (3) and (4), is in general given explicitly by ${ }^{37}$

$$
V_{i j}(p ; \mathbf{R})=\left(4 \pi \varepsilon_{0}\right)^{-1} R^{-3} \exp (i p R)\left\{(1-i p R)\left(\delta_{i j}-3 \hat{R}_{i} \hat{R}_{j}\right)-p^{2} R^{2}\left(\delta_{i j}-\hat{R}_{i} \hat{R}_{j}\right)\right\}
$$


This formula describes the retarded electrodynamical coupling between two components, separated by a displacement $\mathbf{R}$ of length $R$, associated with a transfer between them of energy equal to $\hbar c p$; if zero energy is exchanged, i.e. $p=0$, the electromagnetic coupling can be described as static. The expressions given by equations (4) also involve a generalized transition polarizability tensor, $\boldsymbol{\alpha}_{f i}(\xi)$, defined by equation (5.2.7) of standard ref. 38, representing the capacity of molecule $\xi$ to undergo a two-photon interaction associated with transition from an initial to a final state, $f \leftarrow i$, via energy non-conserving virtual states. Generally - and electronic transition selection rules permitting - all four terms of equation (2) contribute to the quantum amplitude $M^{A}$, as they connect the same initial and final system states.

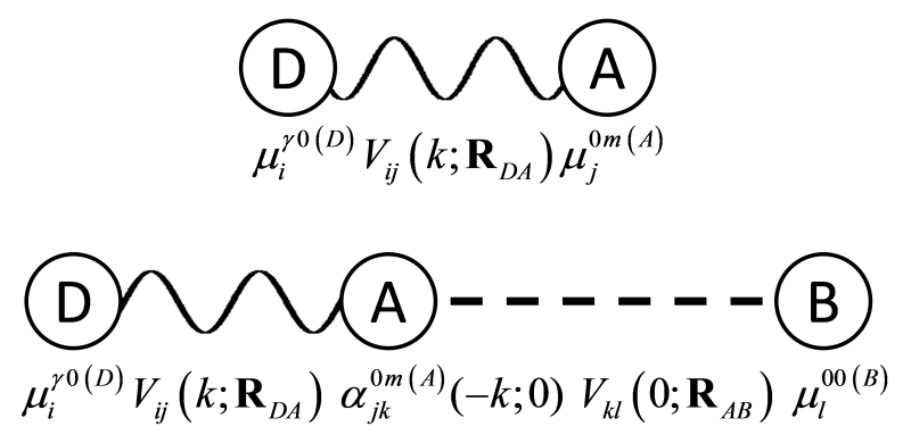

Figure 2. Coupling configurations for energy transfer described by quantum amplitudes $M^{D A}$ and $M^{D A B}$. The coupling between $A$ and $B$ in the latter is static due to $p=0$, illustrated by a dashed line. Two other forms of coupling arise, not depicted here, corresponding to expressions in the second and third lines of equations (4): these are configurations centered on $B$ and on $D$.

Since the square modulus of the entire amplitude $M^{A}$ directly relates to the fluorescence detection signal, cross-terms corresponding to quantum interference between the various contributions will also have physical significance. The leading term is provided by equation (3), and its square modulus is given by;

$$
\begin{aligned}
&\left|M^{D A}\right|^{2}=\left(4 \pi \varepsilon_{0}\right)^{-2} R_{D A}^{-6}\left|\mu^{0 m(A)}\right|^{2}\left|\mu^{\gamma 0(D)}\right|^{2} \\
& \times \mid\left\{\sin \theta \cos \phi \cos \psi\left[\left(1-3 R_{O A}^{2} R_{D A}^{-2}\right)\left(1-i k R_{D A}\right)-k^{2} R_{D A}^{2}\left(1-R_{O A}^{2} R_{D A}^{-2}\right)\right]\right. \\
&+\sin \theta \sin \phi \sin \psi\left[1-k^{2} R_{D A}^{2}-i k R_{D A}\right] \\
&\left.-\cos \theta \cos \psi R_{O D} R_{O A} R_{D A}^{-2}\left[3-k^{2} R_{D A}^{2}-3 i k R_{D A}\right]\right\}\left.\right|^{2} .
\end{aligned}
$$

Here, distances $R_{O D}$ and $R_{O A}$ signify the displacements of the detector and emitter $A$, respectively, relative to the coordinate origin (mid-way between $A$ and $B$ ). The result exhibits an interesting distance-dependence in the near-field region, as shown in the upper graph of Figure 3 ; there is a detector position at $k R_{O D} \approx 0.019$ at which the signal disappears, when the emitters are suitably oriented.

The expression given in equation (6), independent of $B$, will be the leading contribution to the detector signal, followed by quantum interference between $M^{D A}$ and the three fourth-order amplitudes. Which of the latter contributions is the next-leading term will depend upon their relative magnitudes - an issue that is primarily determined by the relative displacements of the components, but also by the particular selection rules for single-photon and two-photon transitions (associated with transition dipole and transition polarizability interactions, respectively).

First, if $A$ and $B$ are chemically different, and their decay transitions are two-photon and single-photon forbidden, respectively, then $M^{D A B}$ and $M^{B D A}$ will vanish. The leading contribution to the detector signal that is sensitive to the relative positioning of $A$ and $B$ is therefore: 


$$
\begin{aligned}
2 \operatorname{Re}\left(\bar{M}^{D A} M^{D B A}\right)= & 2\left(4 \pi \varepsilon_{0}\right)^{-3}\left|\mu^{0 m(A)}\right|^{2}\left|\mu^{\gamma 0(D)}\right|^{2} R_{D A}^{-6} R_{A B}^{-3} \\
& \times \operatorname{Re}\left[\exp \left(i k R_{A B}\right) \Omega T_{j} U_{k} \alpha_{j k}^{00(B)}(-k ; k)\right],
\end{aligned}
$$

where $\Omega=\sin \theta \cos \phi T_{x}+\sin \theta \sin \phi T_{y}-\cos \theta T_{z}$ and the following shorthand is employed:

$$
\begin{aligned}
& T_{x}=\cos \psi\left\{\left(1-3 R_{A B}^{2}\left[R_{A B}^{2}+4 R_{O D}^{2}\right]^{-1}\right)\left(1-i k R_{D A}\right)-k^{2} R_{D A}^{2}\left(1-R_{A B}^{2}\left[R_{A B}^{2}+4 R_{O D}^{2}\right]^{-1}\right)\right\} \\
& T_{y}=\sin \psi\left\{1-k^{2} R_{D A}^{2}-i k R_{D A}\right\} \\
& T_{z}=-\cos \psi\left\{-3+k^{2} R_{D A}^{2}+3 i k R_{D A}\right\} R_{A B} R_{O D}\left[R_{A B}^{2}+4 R_{O D}^{2}\right]^{-1} \\
& U_{x}=\sin \theta \cos \phi\left\{-2+2 i k R_{A B}\right\} \\
& U_{y}=\sin \theta \sin \phi\left\{1-k^{2} R_{A B}^{2}-i k R_{A B}\right\} \\
& U_{z}=\cos \theta\left\{1-k^{2} R_{A B}^{2}-i k R_{A B}\right\}
\end{aligned}
$$

The lower graph of Figure 3 illustrates how the pair-dependent part of the signal also depends on the relative values of the emitter static polarizability components. Notice the correlation of the signal zero with the result for $\left|M^{D A}\right|^{2}$ in the upper graph. The lower graph shows that it is the polarizability components in the $(x, y)$ plane that most significantly contribute to the position-sensitivity of the pair fluorescence signal.

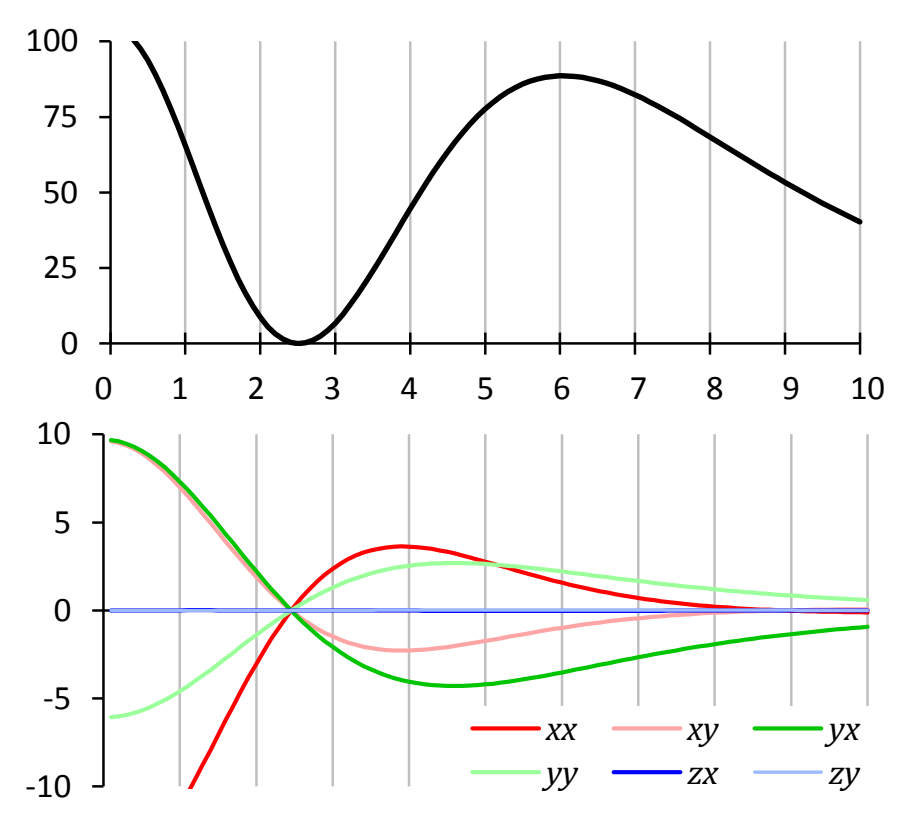

Figure 3. Upper graph: uncoupled fluorescence signal $\left|M^{D A}\right|^{2}$ from equation (6) (units of $100 \varepsilon_{0}^{-2}\left|\mu^{A}\right|^{2}\left|\mu^{B}\right|^{2}$ ) plotted against $125 k R_{O D}$, signifying relative detector displacement. Lower graph: $\operatorname{Re}\left(\bar{M}^{D A} M^{D B A}\right) / \operatorname{Re}\left(\alpha_{i j}^{00(B)}\right)$ from equation (7) (units of $10^{6} \varepsilon_{0}^{-3}\left|\mu^{A}\right|^{2}\left|\mu^{B}\right|^{2}$ ), for six permutations $i j$, again plotted against $125 k R_{O D}$. Here, $R_{A B}=1 / 10 k ; \theta=90^{\circ} ; \phi=\psi=51.7^{\circ}$. 


\subsection{Excitation delocalized across the pair}

Now if $A$ and $B$ are chemically identical, and there is significant electronic coupling between them (without any wavefunction overlap signifying chemical connectivity), then delocalization of the initial excitation across the emitting pair must be considered. In such circumstances, the initial quantum state is correctly described as a superposition of two localized-excitation states, with the appropriate normalization factor included. The relevant combinations, denoted as either symmetric $\left|i^{+}\right\rangle$or antisymmetric $\left|i^{-}\right\rangle$, are defined explicitly by: ${ }^{39}$

$$
\left|i^{ \pm}\right\rangle=2^{-1 / 2}\left|D_{0}\right\rangle\left(\left|A_{m}, B_{0}\right\rangle \pm\left|A_{0}, B_{m}\right\rangle\right)
$$

The nanoemitter pair may undergo fluorescence emission from each of these two exciton states, and the quantum amplitude for the detection of either process is given by;

$$
M_{ \pm}=2^{-1 / 2}\left(M^{A} \pm M^{B}\right)
$$

Each superscript on the right designates the effective position of localized excitation. Moreover, the result for $M^{A}$ is as provided by equation (2), and the chemical similarity (together with the symmetry of this system geometry) implies that the expression for $M^{B}$ takes exactly the same form, with the labels $A$ and $B$ interchanged.
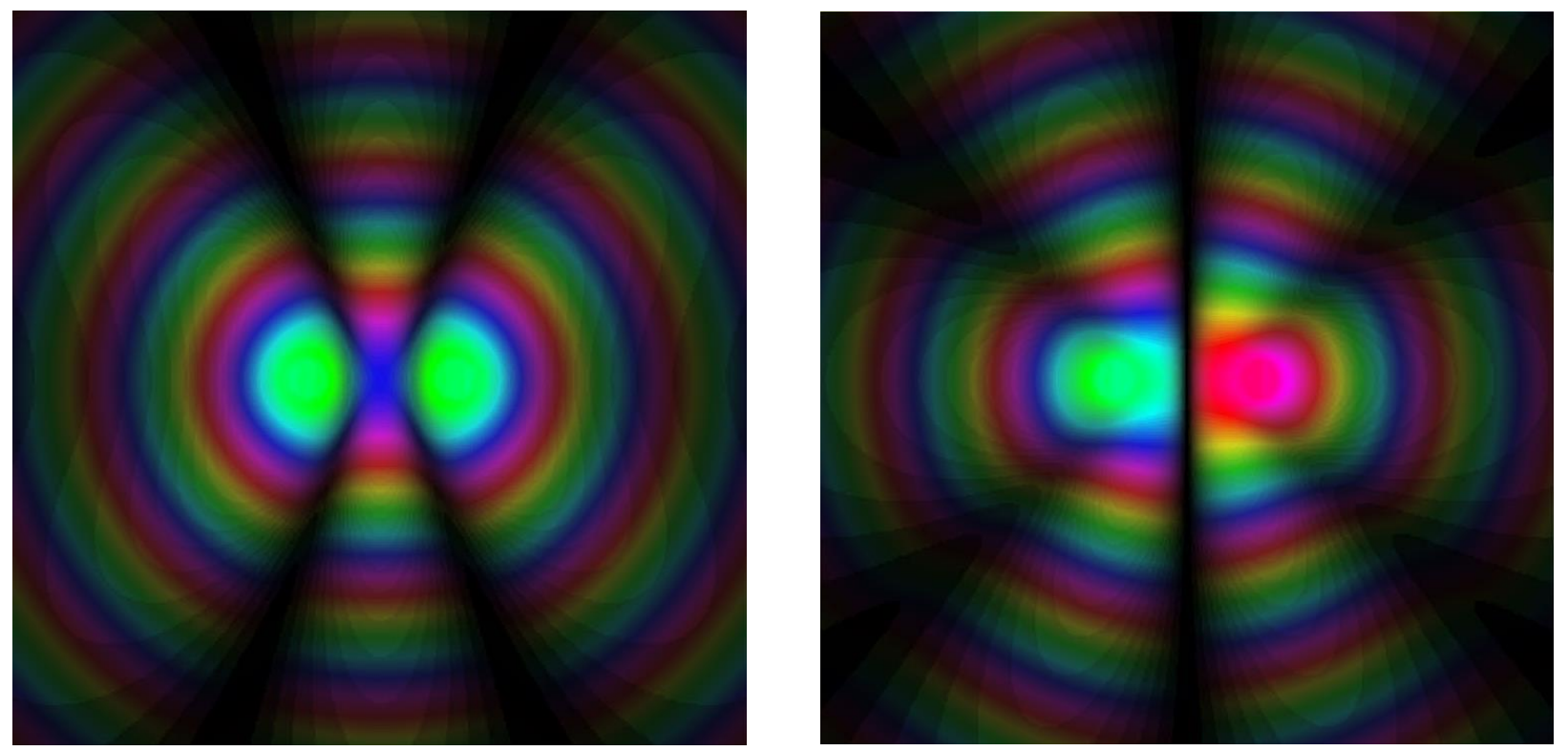

Figure 4: Amplitude (brightness) and complex argument (color hue) of $M_{+}$(left) and $M_{-}$(right), correlating with the phase of the emitted field, for near-field positions of $D$ in the $(x, y)$ plane. Angle $\theta=0$; result averaged over $\psi$, signifying a detector that is insensitive to the orientation of the electric field to which it responds.

The character of the detected signal then depends on the capability of resolving two components in the exciton state. Generally, the coupling of the two emitter states will lead to degeneracy splitting of magnitude $2 \mu_{i}^{0 m(A)} V_{i j}\left(k ; \mathbf{R}_{A B}\right) \mu_{j}^{0 m(B)}$. If the magnitude of this coupling is small, and the emission lines in consequence overlap, the detected emission rate will 
be proportional to $\left(\left|M_{+}\right|^{2}+\left|M_{-}\right|^{2}\right)$, which reduces to the summed result for the independent emitters, i.e. $\left(\left|M^{A}\right|^{2}+\left|M^{B}\right|^{2}\right)$. However, a more interesting case is where the detector is capable of resolving the doublet formed by the symmetric and antisymmetric emission lines. In this instance the associated signals will be determined by $\left|M_{+}\right|^{2}$ and $\left|M_{-}\right|^{2}$ respectively. This leads to the possibility of identifying novel features, which is our focus below.

The present analysis enables us to identify the variation in detected signal as a function of the detector position. There are striking differences in the behavior exhibited by each exciton. Figure 4 exhibits illustrative plots of the fluorescence signal measured by the detector in the near-field, and also the distribution in phase of the detected field, for positions of $D$ in the $(x, y)$ plane. Although both $M_{+}$and $M_{-}$are equal combinations of the two localized-excitation fluorescence amplitudes $M^{A}$ and $M^{B}$, the difference of sign in their combination is equivalent to the introduction of a $\pi$-phase difference between the two emission components, i.e. $M_{-}=2^{-1 / 2}\left(M^{A}+e^{i \pi} M^{B}\right)$. The colors of the brightest regions in Figure 4, nearest $A$ and $B$, exhibit the phases of $M^{A}$ and $M^{B}$, as each localized-excitation component dominates when $D$ is so close to one of the emitters. Moreover, in cases where $\theta=0, \theta=\pi, \phi=\pi / 2$ or $\phi=3 \pi / 2$, then $M_{-}=0$ when $D$ is equidistant between $A$ and $B$. The result, clearly visible in the lower image in Figure 4, is a nodal plane halfway between $A$ and $B$, clearly distinguishing the antisymmetric exciton case.

\subsection{Correlated emission by the pair}

When both nanoemitters are initially excited, transport to the detector of the energy associated with double-excitation decay may occur via another set of processes. The simplest involves a stepwise mechanism, i.e. excitation transfers from $A$ to $D$ and, subsequently from $B$ to $D$. This, of course, requires that the dark time for the detector is less than the interval between the arrival times of the photons conveyed from $A$ and $B$. There is a less demanding but higher order mechanism known as energy pooling, which in a cooperative transfer guise involves the absorption of two photons by the detector at essentially the same time; phenomenologically, this is very similar to the stepwise case. An alternative sub-mechanism is accretive transfer, in which excitation is transferred from one donor to the other before transportation to the acceptor. The theory for each of these mechanisms is equivalent in form to the constructs of equation (4), with appropriate changes to the state designations and $p$ values. In the present situation, since both donors are excited, the sum of the energy absorbed at the two donors is transferred to the acceptor. Much more detail on energy pooling, applied to a different system to those presented here, is found elsewhere. ${ }^{40-42}$

\section{DISCUSSION}

We have shown that the fluorescence emitted by an electronically coupled pair of nanoemitters produces a highly characteristic signal, strongly dependent on the relative positions of the emitters. By specific inclusion of the florescence detector in the calculations, the results are directly tailored for experimental application. In cases where excitation is localized on a single nanoemitter, coupling with a nearby counterpart produces fluorescence with a distinctive dependence on the relative position and orientation of the pair, and on the static polarizability of each emitter. Conversely, when the initial excitation is delocalized across the pair, the emission conveys features characteristic of an exciton doublet. Maps of the detailed intensity and phase distribution then reveal striking departures from the character of single-center emission. ${ }^{43}$ Continuing to pave the way for experimental studies, we now plan to extend this analysis to a more comprehensive study of the collective fluorescence from arrays comprising multiple emitters, including far-field behavior and multiphoton effects.

\section{ACKNOWLEDGMENT}

The authors are thankful to Dr. Kamel Saadi and Matt Coles for the production of Figure 4. The authors would like to thank the Leverhulme Trust and EPSRC for funding this research. 


\section{REFERENCES}

[1] Anger, P., Bharadwaj, P. and Novotny, L., "Enhancement and quenching of single-molecule fluorescence," Phys. Rev. Lett. 96, 113002 (2006).

[2] Novotny, L., "Effective wavelength scaling for optical antennas," Phys. Rev. Lett. 98, 266802 (2007).

[3] Biagioni, P., Huang, J. S. and Hecht, B., "Nanoantennas for visible and infrared radiation," Rep. Prog. Phys. 75, 024402 (2012).

[4] Kühn, S., Håkanson, U., Rogobete, L. and Sandoghdar, V., "Enhancement of single-molecule fluorescence using a gold nanoparticle as an optical nanoantenna," Phys. Rev. Lett. 97, 017402 (2006).

[5] Bakker, R. M., Drachev, V. P., Liu, Z., Yuan, H.-K., Pedersen, R. H., Boltasseva, A., Chen, J., Irudayaraj, J., Kildishev, A. V. and Shalaev, V. M., "Nanoantenna array-induced fluorescence enhancement and reduced lifetimes," New J. Phys. 10, 125022 (2008).

[6] Ringler, M., Schwemer, A., Wunderlich, M., Nichtl, A., Kürzinger, K., Klar, T. A. and Feldmann, J., "Shaping emission spectra of fluorescent molecules with single plasmonic nanoresonators," Phys. Rev. Lett. 100,203002 (2008).

[7] Pellegrini, G., Mattei, G. and Mazzoldi, P., "Light extraction with dielectric nanoantenna arrays," ACS Nano 3, 2715-2721 (2009).

[8] Vecchi, G., Giannini, V. and Gómez Rivas, J., "Shaping the fluorescent emission by lattice resonances in plasmonic crystals of nanoantennas," Phys. Rev. Lett. 102, 146807 (2009).

[9] Curto, A. G., Volpe, G., Taminiau, T. H., Kreuzer, M. P., Quidant, R. and van Hulst, N. F., "Unidirectional emission of a quantum dot coupled to a nanoantenna," Science 329, 930-933 (2010).

[10] Devilez, A., Stout, B. and Bonod, N., "Compact metallo-dielectric optical antenna for ultra directional and enhanced radiative emission,” ACS Nano 4, 3390-3396 (2010).

[11] Mohammadi, A., Kaminski, F., Sandoghdar, V. and Agio, M., "Fluorescence enhancement with the optical biconical antenna," J. Phys. Chem. C 114, $7372-7377$ (2010).

[12] Aouani, H., Mahboub, O., Devaux, E., Rigneault, H., Ebbesen, T. W. and Wenger, J., "Plasmonic antennas for directional sorting of fluorescence emission," Nano Lett. 11, 2400-2406 (2011).

[13] Lee, K. G., Chen, X. W., Eghlidi, H., Kukura, P., Lettow, R., Renn, A., Sandoghdar, V. and Gotzinger, S., "A planar dielectric antenna for directional single-photon emission and near-unity collection efficiency," Nat. Photonics 5, 166-169 (2011).

[14] Shegai, T., Chen, S., Miljkovic, V. D., Zengin, G., Johansson, P. and Kall, M., “A bimetallic nanoantenna for directional colour routing," Nat. Commun. 2, 481 (2011).

[15] Agio, M., "Optical antennas as nanoscale resonators," Nanoscale 4, 692-706 (2012).

[16] Lee, K. G., Eghlidi, H., Chen, X. W., Renn, A., Götzinger, S. and Sandoghdar, V., "Spontaneous emission enhancement of a single molecule by a double-sphere nanoantenna across an interface," Opt. Express 20, 2333123338 (2012).

[17] Rolly, B., Stout, B. and Bonod, N., "Boosting the directivity of optical antennas with magnetic and electric dipolar resonant particles," Opt. Express 20, 20376-20386 (2012).

[18] Stefani, F. D., Vasilev, K., Bocchio, N., Stoyanova, N. and Kreiter, M., "Surface-plasmon-mediated singlemolecule fluorescence through a thin metallic film," Phys. Rev. Lett. 94, 023005 (2005).

[19] Alù, A. and Engheta, N., "Boosting molecular fluorescence with a plasmonic nanolauncher," Phys. Rev. Lett. 103, 043902 (2009).

[20] Li, X., Kao, F.-J., Chuang, C.-C. and He, S., "Enhancing fluorescence of quantum dots by silica-coated gold nanorods under one- and two-photon excitation," Opt. Express 18, 11335-11346 (2010).

[21] Schmelzeisen, M., Zhao, Y., Klapper, M., Müllen, K. and Kreiter, M., "Fluorescence enhancement from individual plasmonic gap resonances," ACS Nano 4, 3309-3317 (2010).

[22] Sorger, V. J., Pholchai, N., Cubukcu, E., Oulton, R. F., Kolchin, P., Borschel, C., Gnauck, M., Ronning, C. and Zhang, X., "Strongly enhanced molecular fluorescence inside a nanoscale waveguide gap," Nano Lett. 11, 4907 4911 (2011).

[23] Batabyal, S., Mondol, T., Das, K. and Pal, S. K., "Forster resonance energy transfer in a nanoscopic system on a dielectric interface," Nanotechnology 23, 495402 (2012).

[24] Zhao, L., Ming, T., Shao, L., Chen, H. and Wang, J., "Plasmon-controlled Förster resonance energy transfer," J. Phys. Chem. C 116, 8287-8296 (2012). 
[25] Bradshaw, D. S., Leeder, J. M. and Andrews, D. L., "Nonlinear optical techniques for improved data capture in fluorescence microscopy and imaging," Proc. SPIE 7571, 75710B (2010).

[26] Leong, K., Chen, Y., Masiello, D. J., Zin, M. T., Hnilova, M., Ma, H., Tamerler, C., Sarikaya, M., Ginger, D. S. and Jen, A. K. Y., "Cooperative near-field surface plasmon enhanced quantum dot nanoarrays," Adv. Funct. Mater. 20, 2675-2682 (2010).

[27] Holmström, P., Yuan, J., Qiu, M., Thylén, L. and Bratkovsky, A. M., "Passive and active plasmonic nanoarray devices," Proc. SPIE 8070, 80700T (2011).

[28] Abramson, J., Palma, M., Wind, S. J. and Hone, J., "Quantum dot nanoarrays: Self-assembly with single-particle control and resolution," Adv. Mater. 24, 2207-2211 (2012).

[29] Osgood, R. M., Stenhouse, P. D., Quigley, C. E., Hoey, M. L., Carlson, J. B., Lopez, K., Kooi, S., Armstrong, L., Haines, C., Kapoor, D. and Cotlet, M., "Large-area Ag nanoparticle arrays for plasmonically enhanced Raman scattering," Proc. SPIE 8457, 845738 (2012).

[30] Ortolani, M., Limaj, O., D'Apuzzo, F., Giliberti, V., Di Gaspare, A., Mattioli, F., Leoni, R., Sennato, S., Domenici, F., Bordi, F. and Lupi, S., "Differential Fano interference spectroscopy of subwavelength hole arrays for mid-infrared mass sensors," Proc. SPIE 8631, 86310J (2013).

[31] Rivera, V. A. G., Ledemi, Y., El-Amraoui, M., Messaddeq, Y. and Marega, E., "Resonant near-infrared emission of $\mathrm{Er}^{3+}$ ions in plasmonic arrays of subwavelength square holes," Proc. SPIE 8632, 863225 (2013).

[32] Evers, J., Kiffner, M., Macovei, M. and Keitel, C. H., "Geometry-dependent dynamics of two Lambda-type atoms via vacuum-induced coherences," Phys. Rev. A 73, 023804 (2006).

[33] Schmid, S. I. and Evers, J., "Dipole-dipole interaction between orthogonal dipole moments in time-dependent geometries," Phys. Rev. A 77, 013822 (2008).

[34] Schmid, S. I. and Evers, J., "Interplay of vacuum-mediated inter- and intra-atomic couplings in a pair of atoms," Phys. Rev. A 81, 063805 (2010).

[35] Rice, E. M. and Andrews, D. L., “Optical emission of a molecular nanoantenna pair,” J. Chem. Phys. 136, 244503 (2012).

[36] Daniels, G. J. and Andrews, D. L., "The electronic influence of a third body on resonance energy transfer," J. Chem. Phys. 117, 6882-6893 (2002).

[37] Andrews, D. L. and Bradshaw, D. S., "Virtual photons, dipole fields and energy transfer: a quantum electrodynamical approach,” Eur. J. Phys. 25, 845-858 (2004).

[38] Craig, D. P. and Thirunamachandran, T., [Molecular Quantum Electrodynamics: An Introduction to RadiationMolecule Interactions], Dover Publications, Mineola, NY (1998).

[39] Kasha, M., Rawls, H. R. and El-Bayoumi, M. A., "The exciton model in molecular spectroscopy," Pure Appl. Chem. 11, 371-392 (1965).

[40] Andrews, D. L. and Jenkins, R. D., "A quantum electrodynamical theory of three-center energy transfer for upconversion and downconversion in rare earth doped materials," J. Chem. Phys. 114, 1089-1100 (2001).

[41] Hettich, C., Schmitt, C., Zitzmann, J., Kühn, S., Gerhardt, I. and Sandoghdar, V., "Nanometer resolution and coherent optical dipole coupling of two individual molecules," Science 298, 385-389 (2002).

[42] Andrews, D. L. and Bradshaw, D. S., "Optically nonlinear energy transfer in light-harvesting dendrimers," J. Chem. Phys. 121, 2445-2454 (2004).

[43] Rice, E. M., Bradshaw, D. S., Saadi, K. and Andrews, D. L., "Identifying the development in phase and amplitude of dipole and multipole radiation,” Eur. J. Phys. 33, 345-358 (2012). 